\title{
REMARKS ON SUBSEQUENCES, SUBSERIES AND REARRANGEMENTS
}

\author{
M. BHASKARA RAO, K. P. S. BHASKARA RAO AND B. V. RAO
}

\begin{abstract}
In this paper we shall evaluate the measure theoretic, as well as topological (Baire category theoretic) complexity of the set of convergent subsequences of a given sequence of real numbers. Similar problems regarding subseries and rearrangements of a given series are also discussed.
\end{abstract}

1. Subsequences of a given sequence. In what follows, $2^{\omega}$ stands for the space of infinite sequences of zeroes and ones equipped with the product of discrete topologies on the one hand and product of $\frac{1}{2}, \frac{1}{2}$ measures on the other hand. $T: 2^{\omega} \rightarrow 2^{\omega}$ is the map $(T \varepsilon)_{i}=1-\varepsilon_{i} . \Omega \subset 2^{\omega}$ is the set of those sequences which have infinitely many zeroes and ones. Note that $\Omega^{c}$ is countable and, hence, $\Omega$ is a $G_{\delta}$ set and is of measure one, which is left invariant by $T$. Note also that $T$ is a homeomorphism and is measure preserving.

A property defined for points of $2^{\omega}$ is said to hold almost everywhere if the set of points for which the property holds is of cofirst category or is of measure one.

Let now $\mathbf{a}=\left(a_{n}\right)$ be a sequence of real numbers. For any $\varepsilon$ in $\Omega$, $\varepsilon \mathbf{a}$ is the subsequence of a given by $\left(a_{n_{k}}\right)$ where $n_{1}<n_{2}<n_{3}<\ldots$, and $\varepsilon_{i}=1$ if and only if $i=n_{k}$ for some $k$.

THEOREM 1. Let $\alpha$ be limit point of a. Then, $A_{\alpha}=\{\varepsilon: \alpha$ is a limit point of $\varepsilon \mathrm{a}\}$ is a cofirst category set and is of measure one.

Proof. $A_{\alpha}=\bigcap_{p} \cup_{n} \cap_{k>n}\left\{\varepsilon: \varepsilon_{k}=1\right.$ and $\left.\left|a_{k}-\alpha\right|<1 / p\right\}$, and, hence, is a Borel set. (We remark at this point that various sets we encounter subsequently can be proved to be Borel by similar arguments.) Further, the set $A_{\alpha}$ is a tail set and, hence, either $A_{\alpha}$ or $\Omega-A_{\alpha}$ is of first category [3, p. 84]. But since $T\left(\Omega-A_{\alpha}\right) \subset A_{\alpha}, A_{\alpha}$ cannot be of first category. The statement regarding measure follows by a similar argument using Kolmogorov's zeroone law [2, p. 201].

Corollary 1. Let $P$ be the set of all limit points of a. Then $A=\{\varepsilon: P$ is the set of all limit points of $\varepsilon \mathbf{a}\}$ is a cofirst category set and is of measure one.

Proof. If $\alpha_{1}, \alpha_{2}, \ldots$ is a countable subset of $P$, which is dense in $P$, then $A=\bigcap_{i} A_{\alpha_{i}}$.

Received by the editors April 11, 1977.

AMS (MOS) subject classifications (1970). Primary 40A05; Secondary 28A35, 54A99. 
Corollary 2. If $\operatorname{Lim} \sup \mathbf{a}=\beta$ and $\operatorname{Lim} \inf \mathbf{a}=\mu$ then, $B=\{\varepsilon$ : $\operatorname{Lim} \sup \varepsilon \mathbf{a}=\beta$ and $\operatorname{Lim} \inf \varepsilon \mathbf{a}=\mu\}$. is a cofirst category set and is of measure one.

COROLlARY 3. If a sequence is not convergent then almost every subsequence is not convergent, that is, if a is not convergent then $C=\{\varepsilon: \varepsilon \mathrm{a}$ is not convergent $\}$ is a cofirst category set and is of measure one.

2. Subseries of a given series. If $\mathbf{s}=\Sigma a_{n}$ is a series of real numbers and $\varepsilon$ is in $\Omega$, then $\varepsilon$ is the series $\Sigma \varepsilon_{i} a_{i}$, or, equivalently, the subseries of $\Sigma a_{n}$ given by $\sum a_{n_{k}}$, where $n_{1}<n_{2}<\ldots$ and $\varepsilon_{i}=1$ if and only if $i=n_{k}$ for some $k$.

THEOREM 2. (i) If $\mathbf{s}$ diverges to $+\infty$ then, in both senses, almost all subseries diverge to $+\infty$.

(ii) If $\mathbf{s}$ diverges to $-\infty$ then, in both senses, almost all subseries diverge to $-\infty$.

(iii) If $\mathbf{s}$ oscillates then, in both senses, almost all subseries oscillate.

Proof is similar to that of Theorem 1 and, hence, is omitted. This theorem gives us the following corollary which should be compared with [4] and Theorems 1.5 and 1.10 of [5].

COROLlary 4. If $\mathbf{s}$ is not convergent then, in both senses, almost all subseries are not convergent.

The question that now arises is whether almost every subseries of a convergent series is convergent. A solution is given by the following theorem.

THEOREM 3. Let $\mathrm{s}=\Sigma a_{n}$ be a convergent series. Then either almost every subseries is convergent or almost every subseries is not convergent. In the measure theoretic sense the first or second case occurs according as $\Sigma a_{n}^{2}<\infty$ or $\Sigma a_{n}^{2}=\infty$. In the category theoretic sense the first or second case occurs according as $\Sigma\left|a_{n}\right|<\infty$ or $\Sigma\left|a_{n}\right|=\infty$.

Proof. The first statement is a consequence of the zero-one laws. The second statement can be derived from Kolmogorov's three series theorem [2, p. 199] as follows: Consider $X_{n}(\varepsilon)=\varepsilon_{n} a_{n}$. Since $\sum a_{n}$ is convergent, $X_{n}$ is a uniformly bounded sequence of random variables with $\Sigma X_{n}$ convergent almost surely if and only if $\Sigma \operatorname{Var}\left(X_{n}\right)=\Sigma E\left(X_{n}^{2}\right)-\left(E X_{n}\right)^{2}<\infty$, that is, if and only if $\Sigma\left(a_{n}^{2} / 2-a_{n}^{2} / 4\right)=\Sigma a_{n}^{2} / 4<\infty$.

The final statement of the theorem will be proved using the following lemma.

LEMMA. Let $X$ be a metric space and $f_{n}$ be a sequence of real continuous functions on $X$.

(i) If $\left\{x: \sup _{n} f_{n}(x)<\infty\right\}$ is of second category then there is a nonempty open set $U$ such that $\sup _{n} \sup _{x \in U} f_{n}(x)<\infty$.

(ii) If $\left\{x: \inf _{n} f_{n}(x)>-\infty\right\}$ is of second category then there is a nonempty open set $U$ such that $\inf _{n} \inf _{x \in U} f_{n}(x)>-\infty$. 
(iii) If $\left\{x: \sup _{n}\left|f_{n}(x)\right|<\infty\right\}$ is of second category then there is a nonempty open set $U$ such that $\sup _{n} \sup _{x \in U}\left|f_{n}(x)\right|<\infty$.

Proof. To prove (i) observe that $B_{k}=\left\{x: \sup f_{n}(x)<k\right\}$ is a closed set and $\cup_{k>1} B_{k}$ is of second category. Other parts are similarly proved.

Now to prove the last part of Theorem 3, for $n \geqslant 1$ define $f_{n}$ on $\Omega$ by $f_{n}(\varepsilon)=\sum_{i<n} \varepsilon_{i} a_{i}$. If almost every subseries is convergent, that is, $\{\varepsilon: \varepsilon s$ is convergent $\}$ is of second category, then by (iii) of the above lemma there is a nonempty open set $U$ such that $\sup _{n} \sup _{x \in U}\left|f_{n}(\varepsilon)\right|=k<\infty$. So there is an $\varepsilon_{0}$ and an integer $m$ such that for any $\varepsilon$ with $\varepsilon(i)=\varepsilon_{0}(i)$ for $i<m$, we have $\sup \left|f_{n}(\varepsilon)\right| \leqslant k$ showing that $\mathbf{s}$ is absolutely convergent. Conversely, if $\mathbf{s}$ is absolutely convergent, then, in fact, every subseries is convergent.

EXAMPLE. In the measure theoretic sense, almost every subseries of $\Sigma(-1)^{n} / n$ is convergent, whereas almost every subseries of $\Sigma(-1)^{n} / \sqrt{n}$ is not. However, in the category sense, for any of these series almost no subseries converges.

3. Rearrangements of series. For a series $\mathbf{s}=\sum a_{n}$ and a permutation $\pi$ of $N=1,2, \ldots$, the series $\sum a_{\pi(n)}$, which is a rearrangement of $\mathbf{s}$, is denoted by $\pi \mathrm{s}$. If $N^{N}$ is given the product of discrete topologies on $N$, then $N$ !-the set of permutations of $N$-regarded as a subset of $N^{N}$ is a topologically complete separable metric group. $N$ is neither locally compact, nor does there seem to exist any naturai measure on it. If a series $\mathbf{s}$ is absolutely convergent, then every rearrangement of $s$ is convergent. Conversely, if every rearrangement of $\mathbf{s}$ is convergent, then $\mathbf{s}$ is absolutely convergent. The following theorem strengthens this statement.

THEOREM 4. If $\{\pi: \pi \mathrm{s}$ is convergent $\}$ is of second category then $\mathbf{s}$ is absolutely convergent.

Proof. Let $\mathbf{s}=\sum a_{n}$ be the given series. For $n \geqslant 1$ define $f_{n}$ on $N$ ! by

$$
f_{n}(\pi)=\sum_{i<n} a_{\pi(i)}
$$

Each $f_{n}$ is continuous, and by (iii) of the lemma of $\$ 2$ we get a nonempty open set $U$ such that

$$
\sup _{n} \sup _{\pi \in U}\left|f_{n}(\pi)\right|=K<\infty .
$$

From the topoology of $N$, this means that there is a $\pi_{0}$ and an integer $m$ such that for any permutation $\pi$ with $\pi(i)=\pi_{0}(i)$ for all $i<m$ we have $\sup _{n}\left|f_{n}(\pi)\right|$ $<K$. Hence $\mathrm{s}$ is absolutely convergent.

From the proof of the theorem we can, in fact, deduce

COROLlary 5. (a) If $\{\pi$ : partial sums of $\pi \mathbf{s}$ are bounded above $\}$ is of second category then $\varepsilon \mathrm{S}=\sum \varepsilon_{n} a_{n}<\infty$, where $\varepsilon_{n}=1$ or 0 according as $a_{n}$ is positive or negative.

(b) If $\{\pi$ : partial sums of $\pi \mathbf{s}$ are bounded below $\}$ is of second category then 
$\varepsilon s=\sum \varepsilon_{n} a_{n}<\infty$, where $\varepsilon_{n}=-1$ or 0 according as $a_{n}$ is negative or positive.

As a consequence we have the following result of Agnew [1].

COROLLARY 6. If $\mathbf{s}$ is conditionally convergent then almost every rearrangement is not convergent. More precisely, almost every rearrangement $\pi \mathrm{s}$ oscillates between $+\infty$ and $-\infty$.

\section{REFERENCES}

1. R. P. Agnew, On rearrangements of series, Bull. Amer. Math. Soc. 46 (1940), 797-799.

2. P. R. Halmos, Measure theory, Van Nostrand, Princeton, N.J., 1950.

3. J. C. Oxtoby, Measure and category, Springer-Verlag, Berlin and New York, 1971.

4. Problem 5805, Amer. Math. Monthly 78 (1971), 679; solution, 79 (1972), 1045.

5. T. Salat, On subseries of divergent series, Mat. Casopis. Sloven. Akad. Vied 18 (1968), 312-338.

Department of Mathematics, University of Sheffield, Sheffield S10, 2TN, England (Current address of M. B. Rao)

Indian Statistical Institute, Calcutta 35, India (Current address of K. P. S. B. Rao and B. V. Rao) 